Artículos

\title{
Exploración de la intención de consumo de la Cascarilla de cacao (Theobroma cacao L.) como infusión: caso Tungurahua-Ecuador*
}

Exploration of the intention to consume the Cocoa husk (Theobroma cacao L.) as an infusion: Tungurahua-Ecuador case Exploração da intenção de consumo da casca de cacau (Theobroma cacao L.) como infusão: o caso Tungurahua, Ecuador

William Fabián Teneda Llerena ${ }^{\mathrm{a}}$

Universidad Técnica de Ambato, Ecuador

wf.teneda@uta.edu.ec

ORCID: http://orcid.org/0000-0003-2245-7717

Maria Dolores Guamán Guevara

Universidad Técnica de Ambato, Ecuador

ORCID: http://orcid.org/0000-0003-4771-6412

Silvia Melinda Oyaque Mora

Universidad Técnica de Ambato, Ecuador

ORCID: http://orcid.org/0000-0002-7278-5201
DOI: https://doi.org/10.11144/Javeriana.cc20-50.eicc

Fecha de recepción: 23 Noviembre 2018

Fecha de aprobación: 12 Junio 2019

Fecha de publicación: 30 Noviembre 2019

\section{Resumen:}

El presente estudio evalúa la intención de consumo de té elaborado a base de la cascarilla de cacao en la provincia de Tungurahua, Ecuador. La cascarilla de cacao (Theobroma cacao L.) es un residuo del proceso de producción del cacao. Estudios recientes han demostrado que este producto tiene propiedades medicinales, lo que potencializa su atractivo de consumo en infusión. Para estimar la demanda se realizó una investigación de mercado a fin de conocer el nivel de aceptación y la disposición de compra de este té, así como determinar estrategias para dar valor agregado al producto. Se encuestaron 386 personas en la provincia de Tungurahua, Ecuador, y fueron identificados 17 productores de chocolate artesanal, quienes desconocen el valor agregado y beneficios de la cascarilla como un producto para bebidas de infusión. La frecuencia de compra del producto correspondió a 15 días con un $47,93 \%$, y es consumido como infusión caliente en un 59,59\%.

Código JEL: D29, L66, M19.

Palabras clave: cacao, cascarilla de cacao, infusión, Theobroma cacao L, chocolate artesanal.

\section{Abstract:}

This study measures the intention of consumption of cocoa husk-based tea in the Tungurahua province, Ecuador. The cocoa husk (Theobroma cacao L.) is a residue of the cocoa production process. Current studies have shown that this product has medical properties, which enhances its consumption appeal. It was made a market research measuring the level of acceptance and the disposition to buy cocoa husk-based tea. This data allows to determine strategies to give value added to the product. 386 people of Tungurahua were surveyed. It was found 17 craft chocolate producers, who do not know the value added and the benefits of using cocoa husk as infusion. The purchase frequency of the product was 15 days with $47,93 \%$, and its consumption as infusion was about $59,59 \%$.

JEL Code: D29, L66, M19.

Keywords: Cocoa, cocoa husk, infusion, Theobroma cacao L, artisanal chocolate.

\section{Resumo:}

O presente estudo avalia a intenção de consumo de chá elaborado a partir da casca de cacau na província de Tungurauha, Equador. A casca de cacau (Theobroma cacao L.) é um resíduo do processo de produção do cacau. Estudos recentes têm demostrado que este produto tem propriedades medicinais, o que potencia seu atrativo de consumo em infusão. Para estimar a demanda realizou-se uma pesquisa de mercado com o objeto de conhecer o nível de aceitação e a disposição de compra deste chá, bem como determinar estratégias para dar valor agregado ao produto. Foram sondadas 386 pessoas na província de Tungurahua, Equador.

Notas de autor:

\footnotetext{
${ }^{a}$ Autor de correspondencia. Correo electrónico: wf.teneda@uta.edu.ec
} 
Nesta província identificaram-se 17 produtores de chocolate artesanal, os quais desconhecem o valor agregado e os benefícios da utilização da casca como um produto para bebidas de infusão. A frequência de compra do produto correspondeu a 15 dias com um 47, 93\%, e é consumido como infusão quente em um 59,59\%.

Código JEL: D29, L66, M19.

Palavras-chave: cacau, casca de cacau, infusão, Theobroma cacao L, chocolate artesanal.

\section{INTRODUCCIÓN}

En la industria de elaboración de productos a base de cacao, después del proceso de secado, fermentado y tostado de las semillas del fruto se genera un residuo denominado cascarilla. Los residuos agroindustriales son productos secundarios obtenidos al procesar cereales, frutas, verduras (Nigam \& Pandey, 2009); se componen de semillas y piel de frutas, verduras que son desechados por desconocimiento o poca valoración de estos (Ayala-Zavala, Gonzalez-Aguilar, \& Siddiqui, 2018).

El cacao fue cultivado y consumido originalmente en América Central. Hace más de 2500 años, los Mayas notaron que la cascarilla poseía propiedades medicinales y terapéuticas. Investigaciones recientes han ratificado que su consumo aporta antioxidantes naturales al cuerpo, los cuales inactivan los radicales libres del proceso de oxidación en el organismo, previene enfermedades cardiovasculares y cancerígenas (Tapia, 2015). Otras concluyen que "los efectos beneficiosos del suplemento de cáscara de cacao, rico en fi bra dietética en pacientes pediátricos con estreñimiento crónico idiopático” (Castillejo \& Millán, 2006). Investigaciones realizadas en Ecuador indican que la "cáscara de cacao" es abundante en magnesio y teobromina, útil para la debilidad, diarrea e inflamación (López, 2013).

En algunos países como Colombia, por ejemplo, los residuos orgánicos son valorados como materia prima porque contienen nutrientes y propiedades. Además, presentan vitaminas, minerales y cáscaras con alta presencia de fibra, semillas pectinas, etc. (Vélez \& Gañan, 2009). La propuesta de que trata esta investigación consiste en darle un valor agregado a la cascarilla de cacao para abrir el mercado de las bebidas de infusión a base de este -considerado hasta ahora- "residuo" de la producción del cacao.

La industria alimenticia es un sector que genera desperdicios orgánicos caracterizados por estar disponibles de forma más o menos permanente en el trópico, o a bajo costo (Maisuthisakul \& Gordon, 2009). Uno de los usos generalizados que se le da a estos desechos es el aprovechamiento para fertilizar el suelo. No obstante, la investigación de Tapia (2015) muestra que el aprovechamiento de subproductos que se obtienen de los desperdicios en la industria alimenticia es un potencial de oportunidades con múltiples usos.

El estudio de Serena (2007) muestra que las industrias tienen alto interés por potenciar el uso de los residuos orgánicos al generar productos que innoven en el mercado. Además que utilizar residuos agroindustriales en la elaboración de productos nuevos es una manera de contribuir al medio ambiente y reducir su impacto al generar alternativas de usos.

\section{PROCESAMIENTO DEL CACAO PARA OBTENER LA CASCARILLA}

En la industria el proceso inicia al limpiar la almendra de cacao fermentada y seca, removiendo las impurezas para garantizar la calidad del producto. Los métodos que se emplean para eliminar agentes contaminantes son: succión para eliminar tierra, piedras livianas, imanes para eliminar metales y vibración para eliminar piedras un poco más pesadas.

Existen dos opciones de tostado y descascarillado; una es el tostado del grano completo (cascarilla y grano); el tostado del grano solamente (luego del descascarillado), este proceso es determinante del sabor característico de los subproductos como el chocolate, remover la humedad y los contaminantes microbiológicos. El pretratamiento térmico consiste en la exposición rápida de la semilla a una fuente de calor intensa como vapor o radiación. 
Solo un 12\% y $8 \%$ del total de la producción es tratado aquí en Ecuador, para comercializarlo como producto elaborado o terminado, lo que contribuye al cambio de la matriz productiva, para incrementar la comercialización y las exportaciones de los diferentes productos ecuatorianos y hacerlos más representativos (Schmid, 2013).

Según la Organización Internacional del Cacao (ICCO por sus siglas en inglés), Ecuador produce el 75\% de cacao fino de aroma, siendo una gran ventaja para productores y asociaciones. La producción anual de cacao fino es de 160 mil toneladas, convirtiéndose en un rubro importante de ingresos para Ecuador. Este tipo de chocolate se distingue por su pureza, sabor y fragancia (MAG, 2017).

En esta industria se emplea los granos o semillas de cacao, que representan un $12 \%$ del fruto, el resto del fruto se descarta, lo que genera problemas ambientales porque al descomponerse emiten olores fétidos; la mayor cantidad de desechos se relaciona con la cáscara los cuales son agentes causantes de plagas en las plantas (Beckett, 2002).

En la economía campesina, el cacao es un producto importante para mejorar los ingresos, se estima que en Latinoamérica un $40 \%$ del presupuesto familiar campesino se financia con la producción y comercialización del cacao, por ello, en muchos países se están implementando proyectos enfocados a incrementar la producción con mejoras en la productividad. Las estadísticas de la Organización de la Naciones Unidas para la alimentación y la agricultura -FAO- del periodo 2001 a 2012 muestran un crecimiento en la producción del 4,5\% anual (Observatorio del caco fino y de aroma para América Latina, 2018). La ICCO recomienda que las variables de sostenibilidad para el cacao se centren en el productor como elemento primordial de la cadena de valor.

Además del cacao en grano, Ecuador exporta semielaborados de cacao; cuyo rubro ha crecido paulatinamente durante los años 2007-2012, al pasar de 8.418,4 toneladas métricas a 23.926, lo que representa un incremento del $184 \%$; asimismo se exporta cacao en grano tostado y cascarilla de cacao (Anecacao, 2015, 2017).

Según la Asociación Nacional de Exportadores de Cacao -Anecacao- las exportaciones en Ecuador alcanzaron las 236 mil toneladas métricas de cacao en grano al finalizar el año5. De esto el $87 \%$ son volúmenes exportados, el $12 \%$ son granos transformados a semielaborados y un $0,8 \%$ corresponde a productos terminados (Anecacao, 2015).

Los productores cacaoteros desechan la cascarilla y algunos semielaborados por desconocer sus propiedades antioxidantes naturales, que son capaces de inactivar los radicales libres del proceso de oxidación del organismo, previniendo la aparición de enfermedades degenerativas, diversos tipos de cáncer, enfermedades cardiovasculares, etc. (Barazarte, 2008). Estas propiedades que mejoran la calidad de vida y satisfacen necesidades de personas que consumen productos por su bienestar (Plan Nacional del Buen Vivir, 2013) se constituye en ventajas para la comercialización de la infusión a base de cascarilla de cacao.

\section{DESCRIPCIÓN DEL PRODUCTO, CARACTERÍSTICAS Y USOS}

Biotea (2017) señala características químicas y los beneficios de la cascarilla del cacao. La cascarilla posee propiedades terapéuticas y medicinales, es abundante en magnesio, ácidos oleicos y linoleico, vitaminas y pectinas. La corteza de cacao posee el alcaloide teobromina con virtudes relevantes para la salud, por beneficiar en cuadros inflamatorios, deficiencia de magnesio, diarreas, astenia.

Cuadros inflamatorios: la teobromina es diurética y anti-inflamatoria, funciona como un tratamiento complementario para paliar la retención de líquidos o edemas en el organismo. Se debe tomar en decocción dos veces al día a razón de una cucharada de corteza de cacao por dosis. 
Deficiencia de magnesio: la decocción de la corteza de cacao ofrece un aporte de este mineral. Sus efectos se obtienen a largo plazo. Puede endulzarse con miel o un poco de azúcar integral de caña para que no resulte excesivamente amarga.

Astenia: la teobromina es un estimulante suave, por lo que resulta un sustitutivo del café, cuya cafeína es un estimulante.

Diarreas: corta las diarreas y recobrar el buen funcionamiento del intestino. Aunque la corteza de cacao es un alimento natural, se debe restringir su consumo en personas que presenten alergias a los derivados del cacao, estreñimiento, acné, hipercolesterolemia grave o dolencias renales (Biotea, 2017).

TABLA 1

Descripción del producto

\begin{tabular}{|c|c|c|}
\hline \multicolumn{3}{|c|}{ Infusión de cascarilla de cacao } \\
\hline Características & Propiedades & Usos \\
\hline \multirow{7}{*}{$\begin{array}{l}\text { Posee propiedades curativas, } \\
\text { diuréticas y anti inflamatorias sobre } \\
\text { diversas patologías básicas }\end{array}$} & $\begin{array}{l}\text { Vitaminas A-C } \\
\text { Rico en fibra }\end{array}$ & $\begin{array}{l}\text { En procesos anti- } \\
\text { inflamatorios }\end{array}$ \\
\hline & Pectina & \\
\hline & Teobromina & Energizante suave \\
\hline & Calcio & \\
\hline & Magnesio & \\
\hline & Ácido oleico y Linoleico & $\begin{array}{l}\text { Puede ser usado como } \\
\text { suplemento nutricional }\end{array}$ \\
\hline & Antioxidantes & \\
\hline
\end{tabular}

Fuente: elaborado por investigadores (Biotea, 2017)

\section{METODOLOGÍA}

Se realizó una encuesta a productores artesanales de Chocolate del cantón Ambato, de acuerdo con datos del gremio artesanal de Tungurahua en el año 2017, son 17 productores de derivados del cacao como el chocolate artesanal (Pasta de Cacao), quienes serían los posibles productores de té de cascarilla para infusiones.

\section{Investigación de Mercado}

Selección del procedimiento del muestreo. Se utilizó un muestreo aleatorio simple, caracterizado por ser un procedimiento de muestreo probabilístico que da la misma probabilidad de ser seleccionado a cada elemento de la población objetivo y a cada muestra de un tamaño determinado.

La población o universo se puede definir como un conjunto de unidades o ítems que comparten algunas notas o peculiaridades que se desean estudiar. La población es una investigación estadística se define arbitrariamente en función de sus propiedades particulares (Hernández, 2001, p. 127). 
La población objeto de estudio la constituyen todas las personas que componen la población económicamente activa de la ciudad de Ambato con un rango de ingresos desde 375 a 700 dólares, lo que corresponde a 89776 personas.

La muestra es una proporción de la población que posee similares características a los objetos o sujetos observados en la población.

$$
n=\frac{z^{2} * p^{*} q^{*} N}{z^{2} * p^{*} q+N * e^{2}}
$$

Dónde:

$\mathrm{n}=$ Número de unidades que componen la muestra

$\mathrm{N}=$ Número de unidades observadas que componen la población

$\mathrm{P}=$ Probabilidad de ocurrencia

$\mathrm{Q}=$ Probabilidad de no ocurrencia

$\mathrm{Z}=$ valor crítico

$\mathrm{e}=$ error

$$
n=\frac{1,96^{2} * 0,5 * 0,5 * 89776}{1,96^{2} * 0,5 * 0,5+89776^{*} 0,05^{2}}
$$

$n=386$

La población meta a investigar asciende a 89.776 de la población económica de la provincia de Tungurahua, la muestra resultante es de 386 personas a las que les aplicó la encuesta.

\section{RESULTADOS Y DISCUSIONES}

En Ecuador, uno de los sectores que más ha crecido en la economía es el sector bebidas dentro de la industria alimentaria, como son: gaseosas, infusiones calientes, tés fríos, jugos, leche y agua. En este orden de ideas, y de acuerdo con datos de (Ramírez \& Araujo, 2016) "señalan que el ecuatoriano promedio consume 150 litros de bebidas y 18 litros de leche al año".

En este contexto y de acuerdo con (Interactuaclub, 2014) las cifras sobre ventas e ingresos, el sector de bebidas tuvo réditos durante el 2014 por 2.645 millones de dólares, lo cual representa el 2\% del PIB del país, los mayores ingresos estuvieron consolidados en el segmento de bebidas gaseosas y otras junto con la cerveza. 
El sector infusiones de té en Ecuador aportan el 2\% del Producto Interno Bruto en conjunto con otras empresas que se dedican al sector bebidas en la economía empero de acuerdo con (Proecuador, 2012) las bebidas calientes tradicionales que prefieren los ecuatorianos son: horchata, té de manzanilla, té de hierba Luisa, té cedrón, té rojo y té negro.

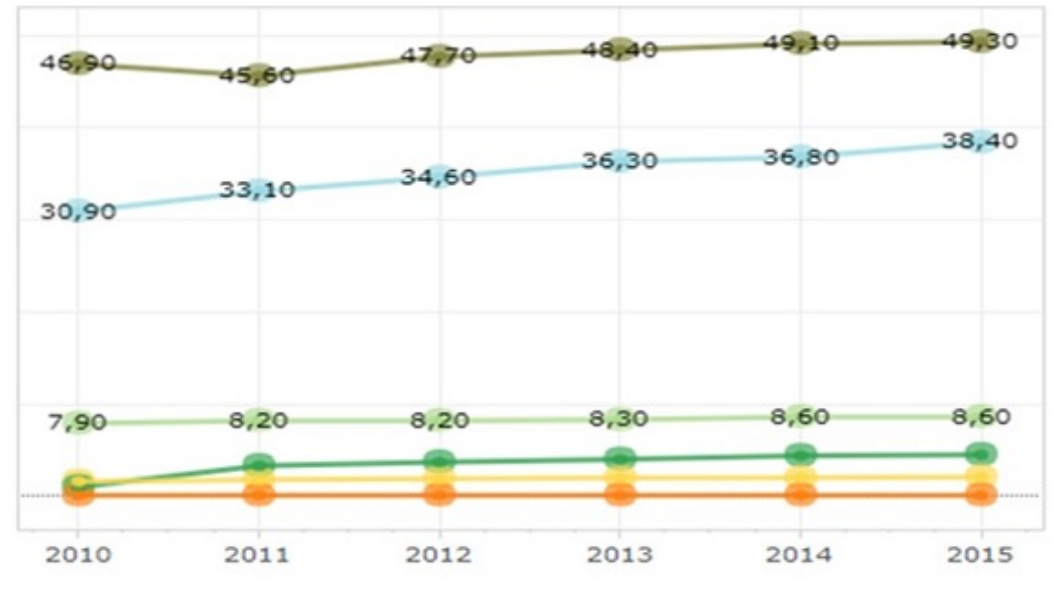

FIGURA 1

Crecimiento del mercado de las bebidas

Fuente: elaborado por Acuamonitor, (Ramírez \& Araujo, 2016).

La figura 1 muestra el comportamiento del mercado de bebidas el segmento infusiones de té caliente y frío, que crecen en un $10 \%$ anual de manera constante. De acuerdo con la asociación de empresas productoras de infusiones de té -AEPT-, Ecuador produce 25000 toneladas de infusiones de té al año.

TABLA 2

Oferta Potencial

\begin{tabular}{ccc}
\hline & Infusión de té caliente o frío & \\
\hline Año & Toneladas & Tasa Crecimiento del 10\% \\
\hline $\mathbf{2 0 1 6}$ & 25000 & 1,1 \\
\hline $\mathbf{2 0 1 7}$ & 27500 & 1,1 \\
\hline $\mathbf{2 0 1 8}$ & 30250 & 1,1 \\
\hline $\mathbf{2 0 1 9}$ & 33275 & 1,1 \\
\hline $\mathbf{2 0 2 0}$ & 36602,5 & 1,1 \\
\hline
\end{tabular}

Fuente: elaboración propia

\section{Canales de Comercialización}

El producto té de cascarilla de cacao, utilizará canales de comercialización a tiendas, comercios minoristas y mayoristas, para su distribución (ver figura 2). La decisión por este tipo de canal de distribución está basada en la aplicación de la encuesta a los propietarios o dueños de negocios mayoristas y minoristas, quienes señalaron que adquieren este tipo de producto para distribuirlas directamente de los productores. 


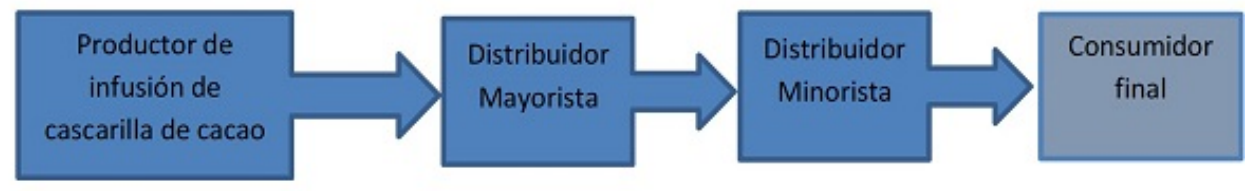

FIGURA 2

Sistema de Comercialización de la infusión de cascarilla de cacao

Fuente: elaboración propia.

\section{Capacidad productiva en Tungurahua}

De acuerdo con datos de la Cámara de Artesanos de la provincia de Tungurahua, Ecuador, en el año 2017 existieron 17 productores que trabajan con derivados del cacao. El 91\% de los productores señalan que los residuos que se obtienen con mayor frecuencia son cascarilla de cacao, mientras que un $9 \%$ señala que los residuos son forrajes. El manejo de residuos o desechos es vital en el desarrollo de las actividades productivas de las empresas chocolateras, tanto por temas de filosofía organizacional, como ambientales, también por el cumplimiento de las ordenanzas municipales, gubernamentales. Sólo, el $6 \%$ de los encuestados manifiestan que los residuos generados en los procesos productivos dentro de su empresa son desechados, mientras que el $76 \%$ señalan que son comercializados, el $18 \%$ restante manifiesta que son usados como alimento para animales de granja.

El manejo adecuado de los residuos sólidos y líquidos de los procesos productivos se constituyen en una parte importante de las estrategias de las empresas en áreas de costos y comercialización. Los ingresos por la comercialización de los residuos no son significativos en los balances económicos de la empresa.

En el estudio con relación a los tipos de cacao que adquieren los productores se tiene que un $71 \%$ de los productores utilizan la variedad de cacao conocida como criollo, porque compran la materia prima en Manabí, el $21 \%$ de los encuestados señalaron que usan la variedad de cacao denominada forastero y el $9 \%$ restante señala que utiliza la variedad de cacao conocido como trinitario.

Para producir cacao sostenible de primera calidad, la Organización de las Naciones Unidas para la Alimentación y la Agricultura (FAO) a nivel internacional trabajan con cooperativas o grupos de productores para facilitar apoyo técnico, acceso a nuevos mercados y mejorar las negociaciones, basados en acuerdos entre gobiernos con el fin de proteger intereses de los productos pequeños (Observatorio del caco fino y de aroma para América Latina, 2018). Las acciones desarrolladas por la FAO permiten mejorar la calidad en toda la línea de producción, generando ingresos que se reinvierten en las localidades beneficiando a campesinos y a la comunidad en general.

El 41\% de los encuestados señalan que su producción mensual va desde 1 a 3 toneladas. El $47 \%$ señala que su capacidad de procesamiento es entre 3 y 6 toneladas métricas, el $6 \%$ señalan que su capacidad instalada les permite procesar entre 6 y 10 toneladas métricas, y el $6 \%$ restante manifiestan que su capacidad instalada le permite procesar más de 10 toneladas métricas. Además, 29\% manifiestan que la maquinaria con las que cuenta la empresa es mecánica, mientras que el $47 \%$ señalan que su maquinaria es electromecánica y un $24 \%$ de los encuestados señalan que su maquinaria es electrónica moderna. Contar con maquinaria y tecnología de última generación garantiza la calidad de los productos elaborados, reduce sistemáticamente los residuos y evita pérdidas tangibles de bienes producidos por errores.

De los 17 productores encuestados señalan que sus empresas generan empleo para 67 personas de los cuales el $47,76 \%$ son de género masculino mientras que el $52 \%$ son de género femenino, quienes demuestran mayor grado de responsabilidad y compromiso. El $24 \%$ de los encuestados señalan que poseen empleados con un rango de edad que va desde 18 a 25 años, mientras que el 35\% señalan que sus colaboradores internos tienen un rango de edad que va desde 25 a los 30 años, el 29\% manifiestan que sus colaboradores internos poseen 
un rango de edad que va de 35 a 40 años, y el 6\% restante señala que posee colaboradores internos mayores de 40 años.

El 17,65\% señala que el volumen de residuos generados por su empresa va desde 30 y 50 kilogramos, el $58,35 \%$ manifiestan que la cantidad de residuos generados por la empresa va entre 50 y 150 kilogramos, mientras que, el $12 \%$ señalan que la cantidad de residuos generados se encuentra entre los 150 y 250 kilogramos, el $12 \%$ restantes que los residuos generados por su empresa generan más de 250 kilogramos de residuos mensualmente. Los residuos generados en los procesos de producción deben ser parte del encadenamiento productivo, debido a que las organizaciones están obligadas a dar respuestas oportunas al cuidado del medio ambiente y a los costos generados por el manejo de los residuos.

Los 17 productores encuestados señalan que los precios del kilogramo de cascarilla de cacao son comercializados alrededor de 0,35 y 0,70 centavos de dólar. Los productores comercializan la cascarilla de cacao, que son residuos del proceso de la extracción de manteca de cacao y la elaboración de la tableta de chocolate amargo, la misma es enviada a la provincia de Guayas a distribuidores.

El cálculo de la capacidad productiva estimada de cascarilla de cacao para la elaboración del té, se obtuvo de promedios arrojados en las fichas de observación de los residuos existentes en el proceso de elaboración del chocolate amargo artesanal.

TABLA 3

Residuos de cascarilla de cacao de acuerdo con la capacidad productiva instalada por los 17 productores de chocolate artesanal

\begin{tabular}{ccccc}
\hline Residuo & $\begin{array}{c}\text { Kilogramos de } \\
\text { producción }\end{array}$ & $\begin{array}{c}\text { Kilogramos de } \\
\text { cascarilla }\end{array}$ & $\begin{array}{c}\text { Promedio de la } \\
\text { capacidad productiva } \\
\text { en kilogramos }\end{array}$ & $\begin{array}{c}\text { Total de } \\
\text { residuos } \\
\text { "Cascarilla" }\end{array}$ \\
\hline $\mathbf{1 2 , 5 0} \%$ & 100 & 12,5 & $4250 \mathrm{~kg}$ & $531,25 \mathrm{~kg}$ \\
\hline
\end{tabular}

Fuente: elaboración propia con base en las encuestas aplicadas.

Los residuos del proceso de elaboración del chocolate artesanal representan el 12,5\% del volumen neto de producción, este valor es el promedio de los dos tipos de cacao que son usados para el procesamiento debido a que la humedad y el $\mathrm{pH}$ no son los mismos. Además, la capacidad productiva promedio de los 17 productores en total es de 4,1 toneladas, que son 4.250 kilogramos de producción mensual generando residuos de 531,25 kilogramos de cascarilla de cacao.

\section{Identificación del mercado consumidor de té de cascarilla de Cacao en la Provincia de Tungurahua}

El 56,48\% de los encuestados pertenecen al género femenino, mientras que el 43,26\% pertenece al género masculino y el $0,26 \%$ de los encuestados no contestan. 
TABLA 4

Características del producto que motiva su compra

\begin{tabular}{lccc}
\hline \multicolumn{1}{c}{ Opciones } & $\begin{array}{c}\text { Frecuencia } \\
\text { Absoluta }\end{array}$ & Frecuencia Relativa & $\begin{array}{c}\text { Frecuencia Relativa } \\
\text { Acumulada }\end{array}$ \\
\hline Sabor & 104,91 & $27,19 \%$ & $27,19 \%$ \\
\hline Propiedades & 109,91 & $28,49 \%$ & $55,68 \%$ \\
\hline Marca & 88,25 & $22,87 \%$ & $78,55 \%$ \\
\hline Presentación & 82,75 & $21,45 \%$ & $100,00 \%$ \\
\hline Total & 386 & $78,55 \%$ & $100,00 \%$ \\
\hline
\end{tabular}

Fuente: elaboración propia con base en las encuestas aplicadas

El 27,19\% de los encuestados realizaría su compra por el sabor como característica principal, el 28,49\% señala que basa su opción de compra por las propiedades que posee cada infusión y su relación con las costumbres y creencias, el $22,87 \%$ señala que por la marca, y el $21,45 \%$ señalaron que decidirían por la presentación. La frecuencia de compra sería semanal 18,65\%, mientras que el 47,93\% de los encuestados señalaron que realizan sus compras cada quince días y el porcentaje restante $33,42 \%$ lo realizan de forma mensual.

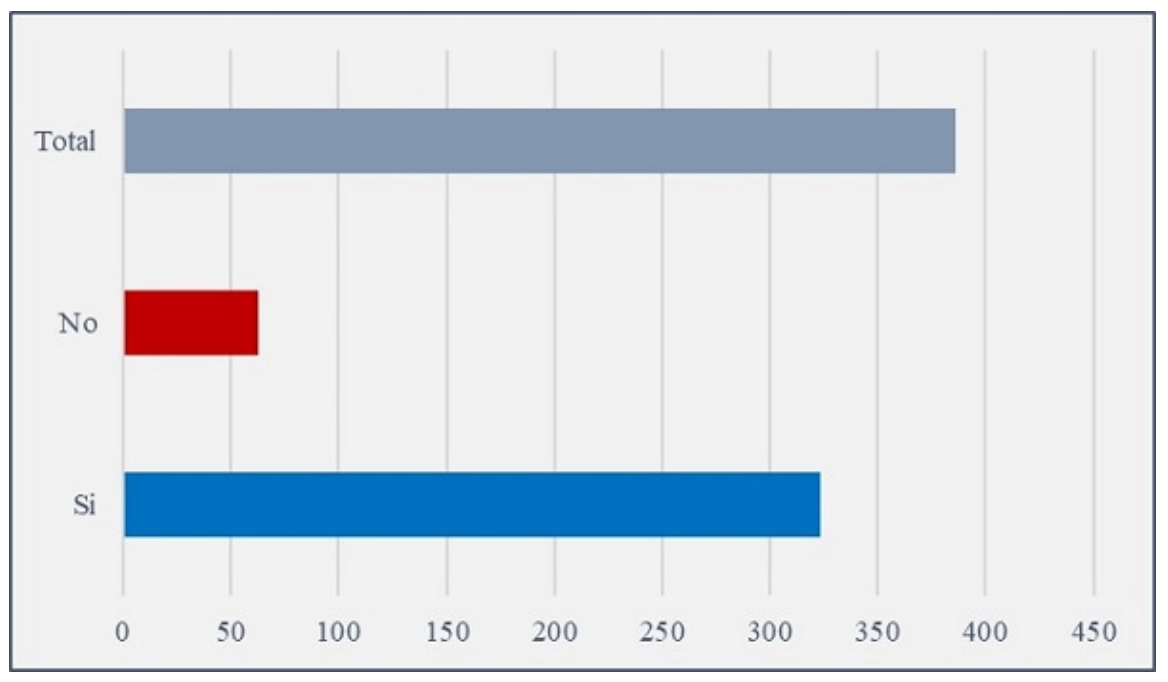

FIGURA 3

Disposición a comprar el té de cascarilla de cacao

Fuente: elaboración propia con base en las encuestas aplicadas.

El 83,68\% de los encuestados señalaron que están dispuestos a comprar té de cascarilla de cacao, mientras que el 16,32\% de los encuestados señalan que no comprarían el té a base de cascarilla de cacao. (En la figura 3 se muestran la comparación de valores absolutos). Y el 22,54\% de los encuestados señalan su preferencia por té frío, mientras que el $77,46 \%$ señala que compran té para infusiones calientes (figura 4). 


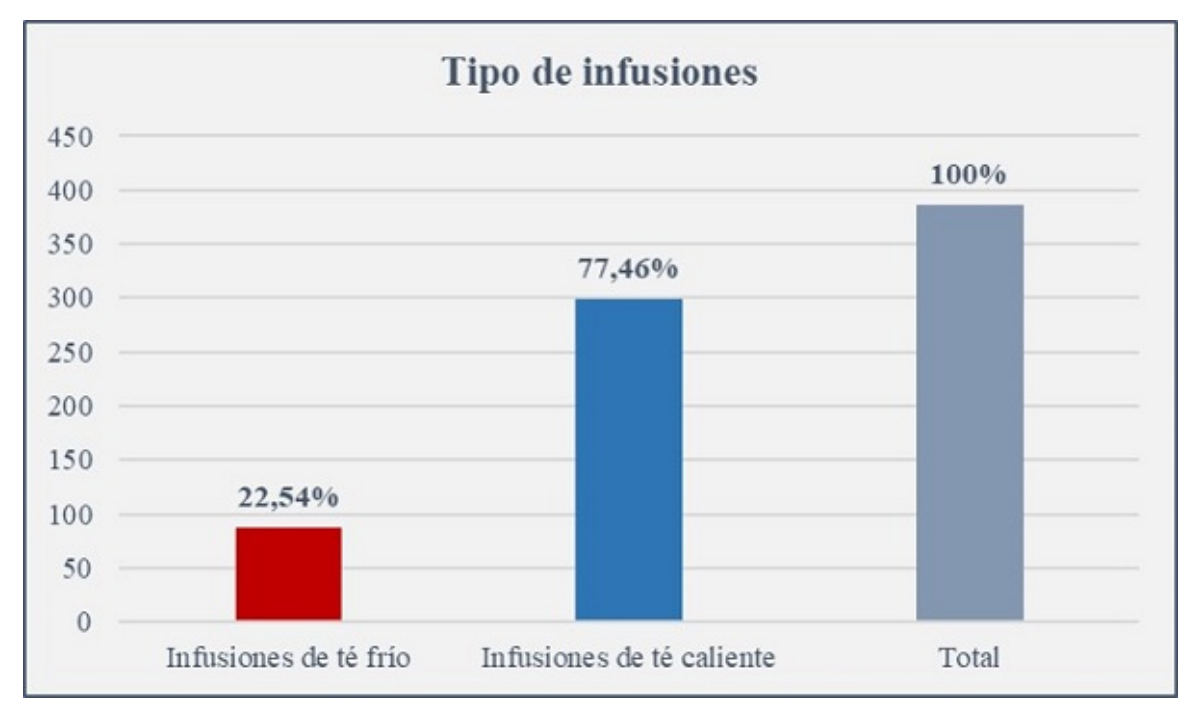

FIGURA 4

Tipos de infusiones

Fuente: elaboración propia con base en las encuestas aplicadas

TABLA 5

Precio de la caja de té

\begin{tabular}{lccc}
\hline \multicolumn{1}{c}{ Opciones } & $\begin{array}{c}\text { Frecuencia } \\
\text { Absoluta }\end{array}$ & $\begin{array}{c}\text { Frecuencia } \\
\text { Relativa }\end{array}$ & $\begin{array}{c}\text { Frecuencia } \\
\text { Relativa } \\
\text { Acumulada }\end{array}$ \\
\hline Caja de bolsas de té de 12 unidades a $\$ 1,1$ & 230 & $59,59 \%$ & $59,59 \%$ \\
\hline Caja de bolsas de té de 12 unidades a $\$ 1,2$ & 119 & $30,83 \%$ & $90,41 \%$ \\
\hline Caja de bolsas de té de 12 unidades a \$ 1,25 & 29 & $7,51 \%$ & $97,93 \%$ \\
\hline Caja de bolsas de té de 12 unidades a \$ 1,3 & 8 & $2,07 \%$ & $100,00 \%$ \\
\hline Total & 386 & $100,00 \%$ & $100,00 \%$ \\
\hline
\end{tabular}

Fuente: elaboración propia con base en las encuestas aplicadas

De los encuestados el 59,59\% señalan que estarían dispuestos a comprar las cajas de doce unidades de bolsitas de infusión de té de cascarilla de cacao a un precio no mayor de 1,10 dólares americanos, mientras que $30,83 \%$ señalan que estarían dispuestos a comprar el producto por un precio de 1,20 dólares americanos, el 7,51\% de los encuestados están dispuestos a pagar un valor hasta ,25 dólares americanos. Y el 2,07\% de los encuestados estarían dispuestos a comprar a un precio no mayor a 1,30 dólares americanos.

\section{CONCLUSIONES}

Los productores de cacao requieren conocimiento, capacidad de innovación, y motivación para iniciar en procesos de reutilización y valoración de residuos orgánicos, con el fin de formar parte de una sociedad responsable, comprometida con el fin de generar incentivos monetarios y no monetarios, y, estar inmerso en políticas ambientales que beneficien su desarrollo (Lett, 2014).

El mercado de bebidas de infusiones de té caliente y frío crecen $10 \%$ anual de manera constante, genera oportunidades en negocios de este tipo. El 76\% de los residuos generados dentro de la elaboración del chocolate artesanal (cascarilla de cacao) son comercializados. El mayor aporte del trabajo productivo en esta cadena productiva le corresponde al género femenino y quienes comprenden una edad entre los 25 y 30 años. 
La frecuencia de compra con mayor determinación corresponde a 15 días, y un alto porcentaje (59,59\%) los consumen como infusión caliente. Los beneficiarios de un té hecho de la cascarilla de cacao serán personas que se preocupan por su salud, además de proveer vitaminas y compuestos que mejoran la salud de forma natural.

\section{Referencias}

Anecacao. (2015). Exportación Ecuatoriana de Cacao, 2015. Asociación Nacional de Exportadores de Cacao, Ecuador. Documento em línea http://www.anecacao.com/uploads/estadistica/resumen-exportacion-de-cacao-2015anecacao-ecuador_1.pdf

Anecacao. (2017). Asociación Nacional de Exportadores de Cacao, Ecuador. Retrieved from http://www.anecacao.com/ es/quienes-somos/cacao-nacional.html

Ayala-Zavala, Gonzalez-Aguilar, \& Siddiqui., W. (2018). Plant Food By-Products. Apple Academic Press.

Barazarte, H. (2008). La cáscara de cacao. Una posible fuente comercial de pectinas. Archivos latinoamericanos de Nutrición, 64-70.

Beckett, S. (2002). La ciencia del Chocolate. En S. Beckett. La ciencia del Chocolate (p. 216). Madrid: Acribia.

Biotea. (2017, Febrero 15). Retrieved from http://www.biotea.es/infusiones/specials/infusion-ecologica-de-cortezade-cacao

Castillejo, G., \& Millán, S. (2006). Estudio controlado, randomizado, a doble estudio ciego, evaluando el efecto de un suplemento de cáscara de cacao rico en fibra sobre el tránsito colónico en pacientes pediátricos con constipación. Nutrición Hospitalaria. Quito.

Hernández, B. (2001). Técnicas Estadísticas de función social. Madrid: Diaz de Santos.

Interactuaclub. (2014). Retrieved from http://www.interactuaclub.com/Blog/Post/el-mercado-de-bebidas-enecuador-para-2016-75

Lett, L. (2014). Las amenazas globales, el reciclaje de residuos y el concepto de economía circular. Revista Argentina de Microbiología, 1-3.

López, P. (2013). Elaboración de compost a partir de cascarilla de cacao. Riobamba: Escuela Superior Politécnica de Chimborazo.

MAG. (2017, febrero). Ministerio de Agricultura y Ganadería. Retrieved from Ministerio de Agricultura y Ganadería: https://www.agricultura.gob.ec/icco-reconoce-el-liderazgo-del-cacao-fino-de-aroma-en-el-mundo/

Maisuthisakul, P., \& Gordon. (2009). Antioxidant and tyrosinase inhibitory activity of mango seed kernel by product. Food Chemistry, 332-341.

Plan Nacional del Buen Vivir. (2013). Plan Nacional del Buen Vivir. Quito.

Nigam, S. \& Pandey, A. (2009). Biotechnology for agro-industrial. Residues utilization. United Kingdom: Springer.

Observatorio del cacao fino y de aroma para América Latina. (2018, Julio). Retrieved from Observatorio del caco fino $y$ de aroma para América Latina: http://scioteca.caf.com/bitstream/handle/123456789/1258/ OLC_CAF_boletin_3_Espa\%C3\%B1ol-final.pdf?sequence=1\&isAllowed=y

PROECUADOR. (2012). Retrieved from http://www.proecuador.gob.ec/wp-content/uploads/2015/02/ PROECU_PPM2012_CACAO_ALEMANIA.pdf

Ramírez, S., \& Araujo, A. (2016, Junio 6). El comercio, p. 4A.

Schmid, P. (2013). Análisis de la situación actual y perspectivas del cacao ecuatoriano y propuesta. Quito: Universidad Internacional del Ecuador.

Serena, A. (2007). Chemical and physicochemical characterisation of co-products from the vegetable food and agro industries. Animal Feed Science and Technology, 139(1), 109-124.

Tapia, C. (2015). Aprovechamiento de residuos agroindustriales. Ambato: Uiversidad Técnica de Ambato.

Vélez, L., \& Gañan, P. (2009). Aprovechamiento de la fibra dietaria y/o residuos de su transformación. Facultad de Ciencias Agropecuarias, 102-104. 


\section{Notas}

* Artículo de investigación científica.

Licencia Creative Commons CC BY 4.0 